\title{
Industrietaugliches Biofilmbildung-Monitoring mit robustem, multiparametrischem Sensorsystem
}

\author{
M. Ruhnow ${ }^{1}$, J. Kohser ${ }^{1}$, M. Bulst ${ }^{2}$, S. Wegner ${ }^{2}$, T. Bley ${ }^{1}$, E. Boschke ${ }^{1}$ \\ ${ }^{1}$ TU Dresden Institut für Lebensmittel- und Bioverfahrenstechnik, Dresden \\ ${ }^{2}$ Sciospec Scientific Instruments GmbH, Bennewitz OT Pausitz
}

\begin{abstract}
:
Biofilme stellen eine ernsthafte gesundheitliche Gefährdung dar, vor allem wenn sie unkontrolliert abgelöst werden [1]. Ziel des vorgestellten Forschungs- und Entwicklungsprojekts zwischen der Sciospec Scientific Instruments $\mathrm{GmbH}$ und der Professur für Bioverfahrenstechnik (TU Dresden) ist daher die Entwicklung, Realisierung und Etablierung eines multiparametrischen Sensorsystems zur Überwachung der Biofilmbildung sowohl im Labormaßstab als auch in industriellen Anlagen. Das Hauptaugenmerk liegt auf einer Kombination von verschiedenen Messprinzipien, um Querempfindlichkeiten oder Störeinflüsse zu minimieren.

Um die erhaltenen Messsignale zur Biofilmbildung interpretieren zu können, eignen sich fluoreszenzmikroskopische Tests. Eine Eigenfluoreszenz der Goldoberfläche der QCM-Chips konnte bei den gewählten Wellenlängen ausgeschlossen und die primäre Adhäsion von E. coli nachgewiesen werden. Vorbereitend für die nachfolgenden Experimente mit den Setups erfolgte die Optimierung der Kultivierungsbedingungen im Bioreaktor.
\end{abstract}

Key words: Biofilm, QCM, multiparameterisches Sensorsystem, Fluoreszenzmikroskopie, primäre Adhäsion.

\section{Motivation}

Mikroorganismen haben das Bestreben, an Oberflächen zu adhärieren und Biofilme zu bilden [2]. Ernsthafte gesundheitliche Schädigungen können durch deren unkontrolliertes Ablösen entstehen. Das ist besonders kritisch beispielsweise im Trinkwasser oder in der Lebensmittelproduktion. Keime gelangen so in die Nahrungskette [1, 3]. 2005 wurden in Deutschland 52.000 Infektionen mit meldepflichtigen Erregern registriert, die sich auf kontaminierte Lebensmittel zurückführen ließen [4]. Für die entsprechenden Unternehmen bedeutet das neben Imageverlust hohe finanzielle Ausfälle z. B. aufgrund der erforderlichen Rückrufaktionen für die betroffenen Produkte [5].

Deshalb sind in Rohrleitungssystemen und Anlagenteilen die Detektion und Überwachung der Bildung von Biofilmen essentiell für eine Vielzahl industrieller Prozesse, auch um vorsorgliche, aufwendige Reinigungsroutinen einzuschränken.

\section{Stand der Technik}

Derzeit, teilweise erst als Forschungsansätze zur Verfügung stehende Messprinzipien zum
Biofilm-Monitoring in geschlossenen Systemen sind [6]:

Detektion von Ablagerungen auf einer Oberfläche

(i) mit Streulicht (OptiQuad vertrieben von OptoSense), (ii) über die Veränderungen im Wärmeübergang, (iii) mit Hilfe von piezoelektrischen Effekten (Vibration, QCMGeräte von Q-Sense) und (iv) dem Messen der Geschwindigkeit von Ultraschallwellen (PULSE III erhältlich bei Ashland Inc.)

Summeninformationen zu den biogenen Komponenten von Ablagerungen

(i) mittels Abgreifen von Veränderungen im Redoxpotential, (ii) Abweichungen in der Fluoreszenz (Optiquad von OptoSense) und (iii) mit der FTIR-ATR-Spektroskopie (FTIRATR)

\section{Tiefergehende Informationen}

(i) mittels Raman Spektroskopie und (ii) Röntgenphotoelektronenspektroskopie 
Die genannten Messsysteme werden zu oft unvertretbar hohen Preisen angeboten. So belaufen sich z. B. die Kosten für das auf Basis von QCM arbeitende Gerät von Q-Sense auf über $50.000 €[6]$. Zudem verfügen die Geräte oft nicht über die notwendigen Kommunikationsschnittstellen. Eine Integration in Industrieautomationen ist somit nicht oder nur sehr schwer möglich.

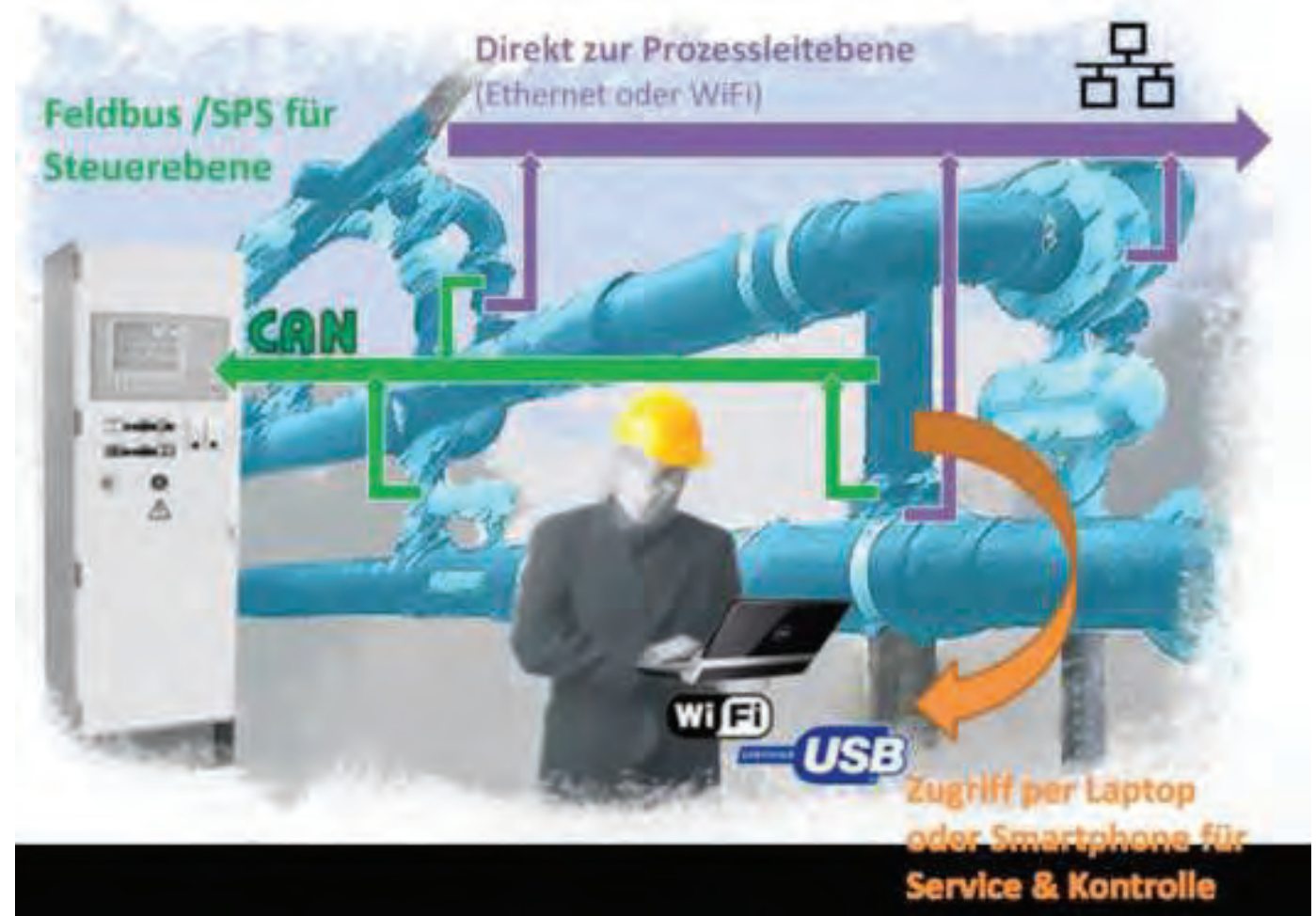

Abb. 1: Prinzipskizze zur geplanten Einbindung des Sensorsystems in industrielle die Prozessautomatisierung.

\section{Technologische Maßnahmen}

Aus den Anforderungen des Marktes und dem aktuellen Angebot an Messlösungen für ein online Monitoring der Biofilmbildung ist abzuleiten, dass der Bedarf an Industrietauglichen Sensorsystemen sehr groß ist. Spezielles Augenmerk liegt dabei auf deren Integrierbarkeit in bestehende industrielle Automatisierungsanlagen (Abbildung 1).

Vor diesem Hintergrund ist das vorgestellte Projekt angesiedelt. Für eine unkomplizierte Prozesseinbindung wird das System im Gegensatz zu verfügbaren Biofilm-Sensoren (s. o.) mit diversen Schnittstellen ausgestattet, wie sie in modernen Automatisierungsanlagen Verwendung finden (CAN, Ethernet, USB, WLAN).

Konkret wird das Sensorsystem folgende Messprinzipien in sich vereinigen: (i) Adhäsionsmessungen mit einer Quarzkristall- mikrowaage ${ }^{1}$, (ii) Bestimmung der elektrischen Leitfähigkeit, (iii) Ermittlung der Kapazität, (iv) Messung der mikrobiell induzierten Trübung und (v) Bestimmung von Wärmeübergangskoeffizienten.

\footnotetext{
${ }^{1}$ Die Quarzkristallmikrowaage (QCM) ist in der Lage, kleinste Masseänderungen zu erfassen. Das dabei verwendete Messprinzip beruht auf dem piezoelektrischen Schwingquarz. Der beidseitig mit Elektroden beschichtete Quarzsensor wird durch das Anlegen einer Wechselspannung in eine resonante Schwingung versetzt. Die dabei entstandene Resonanzfrequenz wird mit einem Impedanzanalysator erfasst. Durch die Anlagerung von Masse, wie es beispielsweise bei der Biofilmbildung stattfindet, kommt es zu einer Dämpfung der Resonanzfrequenz. Basierend auf der Stärke der Änderung dieses Messsignal ist es möglich, die Masseanlagerung zu verfolgen [7].
} 
Die herausragende Neuerung liegt im Konzept der komplexen und multiparametrischen Erfassung und Kompensation der Querempfindlichkeiten. Dies ist ein Ansatz, der derzeit so noch nicht für die Biofilmdetektion verfolgt wird.

\section{Materialen und Methoden}

Das verwendete Bakterium ist Escherichia coli SM2029 (Genotype: ara, (lac-pro), thi attb::blaPa1/04/03-gfp*-T0/pOX38km traD411) [8]. Die allgemeinen Kultivierungsbedingungen sind analog zu der Publikation von Wagner 2013 [9]. Die Kultivierung fand in einem 3 L-Reaktor von Applikon statt. Für die mikroskopischen Untersuchungen wurde ein Axioskop 2 imaging von Zeiss verwendet.

\section{Auswertung}

In Abbildung 2 sind Zellen von Escherichia coli nach einer Stunde stationärer Inkubation bei $30{ }^{\circ} \mathrm{C}$ auf einem QCM-Chip dargestellt. (In Vorversuchen konnte eine Eigenfluoreszenz QCM-Chips ausgeschlossen werden.) Die Kultivierung im Reaktor erfolgte unter konstanten Bedingungen $\left(30^{\circ} \mathrm{C}\right.$, bei $500 \mathrm{rpm}$ ). Die Datenaufzeichnung fand kontinuierlich mit dem Biacontroller von Applikon bzw. dem
Fermenter Control der Firma Optek statt. Es zeigte sich der erwartete Zusammenhang zwischen der Sauerstoffsättigung und der optischen Dichte (OD): mit steigender OD und damit steigender Zellzahl sinkt die Sauerstoffkonzentration ab. Sie steigt wieder an, wenn die stationäre Wachstumsphase erreicht ist (Vergleich Abbildung 3).

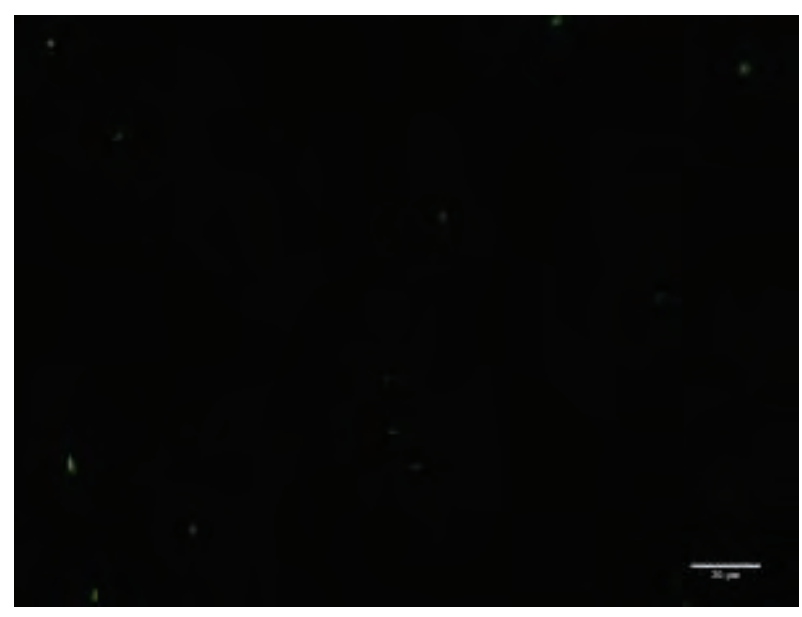

Abb. 2.: Fluoreszenzaufnahme von SM2029 im Axioskop 2 imaging von Zeiss.

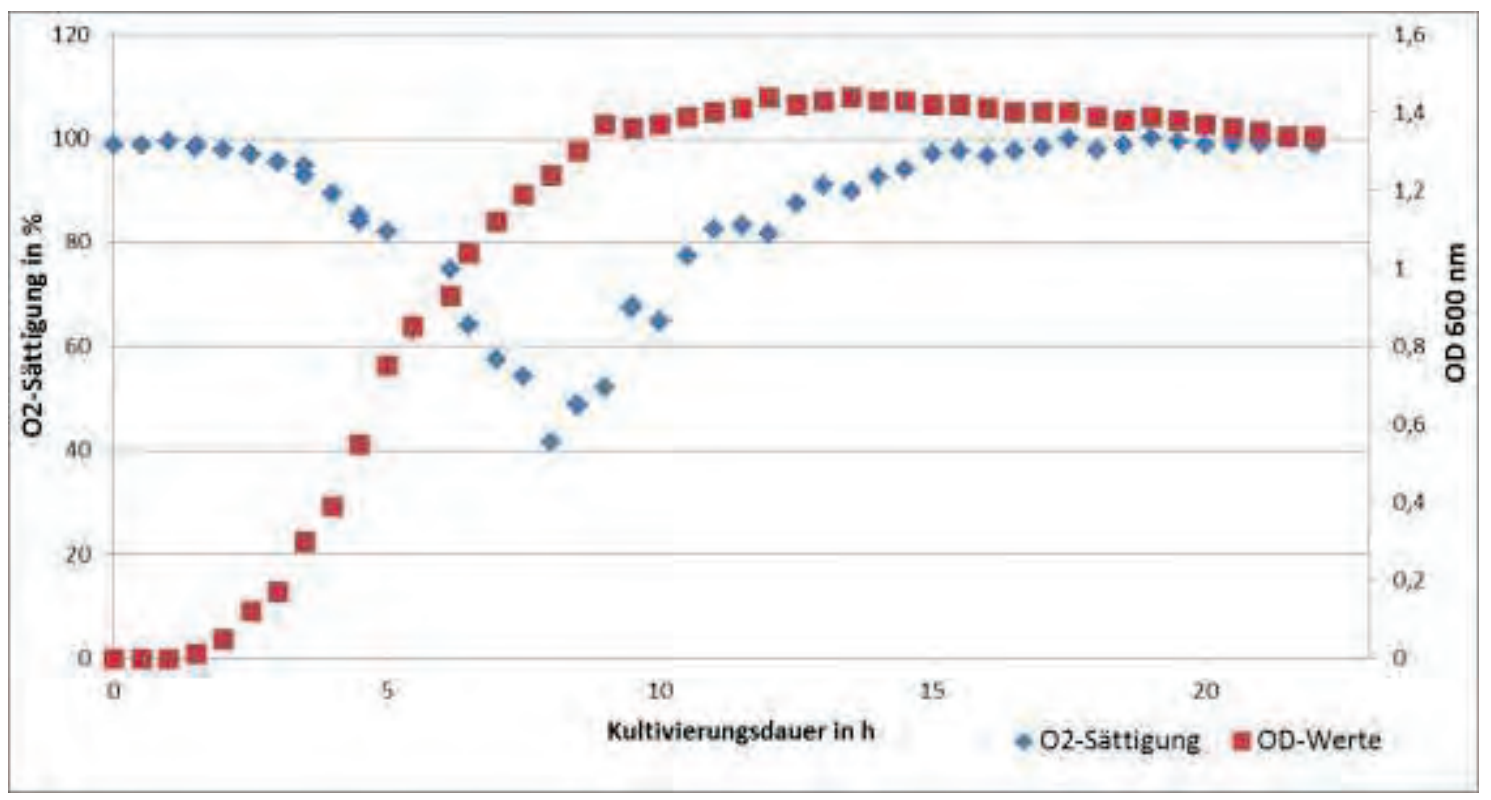

Abb. 3.: Kultivierungsverlauf von Escherichia coli SM2029 im 3 L-Reaktor von Applikon. Es wurde Komplexmedium (LB-Medium) für die Kultivierung verwendet. Die Sauerstoffsättigung konnte mit dem Biacontroller von Applikon, die OD mit Hilfe des Fermenter Controls von Optek kontinuierlich über den gesamten Messverlauf detektiert werden. 


\section{Ausblick}

Die Sciospec $\mathrm{GmbH}$ konzipiert und konstruiert die verschiedenen Sensor-Setups. Sie werden anschließend an der TU Dresden im 3 LBioreaktor und in einer Durchflusskammer getestet, wobei nach erfolgter Nährmedienoptimierung eine geringe Kultivierungsdauer bei gleichbleibender Biofilmbildung angestrebt wird.

In enger Kooperation erfolgt aus den Ergebnissen das Ableiten von Algorithmen zur Fehlerkompensation und Modellen der multiparametrischen Kennlinienfelder.

$\mathrm{Zu}$ Projektende wird das Sensorsystem unter realen Industriebedingungen getestet.

Als Resultat des Projektes ist somit die Erstellung einer umfangreichen Wissensbasis zur Biofilmbildung und deren robuster Messung zu erwarten.

Neben dem wirtschaftlichen Mehrwert, der sich zweifelsohne aus der Biofilmdetektion selbst ergibt, kann die Ausweitung auf andere Sensorsysteme zusätzliche Anwendungsfelder in der Prozessmesstechnik generieren, für die es bislang nur Laborlösungen gibt. Die Überwachung und Steuerung von Reinigungsprozessen stellt nur eine von zahlreichen, viel versprechenden Möglichkeiten für eine spätere technologische Weiterentwicklung dar.

\section{Danksagung}

Die Autoren danken dem Bundesministerium für Wirtschaft und Technologie (BMWi), das das Projekt im Rahmen des Zentralen Innovationsprogramm Mittelstand (ZIM)

\section{Literatur}

[1] L. G. Gutwein, M. Panigrahi, G. S. Schultz und B. A. Mast, „Microbial Barriers," Clinics in Plastic Surgery, Nr. 39, pp. 229-238, 2012.

[2] R. A. N. Chmielewsk und J. F. Frank, "Biofilm Formation and Control in Food Processing Facilities," Comprehensive Reviews in Food Science and Food Safety, Nr. 284, pp. 22-32, 2003.

[3] S. Srey, I. K. Jahid und S.-D. Ha, „Biofilm formation in food industries: A food safety concern," Food Control, Nr. 31, pp. 572-585, 2013.

[4] K. Alpers, „Lebensmittelassoziierte Ausbrüche durch meldepflichtige Erreger in Deutschland 2005," Epidemiologisches Bulletin, Nr. 41, pp. 356-357, 2006.

[5] A. G. J. Velthuis, M. W. Reij, K. Baritakis, M. Dang und C. P. A. van Wagenberg, „Recall costs balanced against spoilage control in Dutch custard," Journal of Dairy Science, Nr. 93, pp. 2779-2791, 2010.

[6] H. C. Flemming und M. Strathmann, „Online sensors for fouling monitoring," Frankfurt am Main, 2010.

[7] I.-K. Lee „In-situ Prozessanalyse der CVSSynthese von nano-kristallinem Siliziumkarbid mit Hilfe eines Aerosolmassenspektrometers." Cuvillier Verlag, S. 38-42, 2004.

[8] A. Reisner, J. A. J. Haagensen, M. A. Schembri, E. L. Zechner et al., "Development and maturation of Escherichia coli K-12 biofilms. " Mol. Microbiol., Nr. 48, pp. 933-946, 2003.

[9] K. Wagner, S. Friedrich, C. Stang, T. Bley et al. "Initial phases of microbial biofilm formation on opaque, innovative anti-adhesive surface using a modular microfluidic system." Eng. Life Science, DOI: 10.1002/elsc.201200035, 2013. 